\title{
Epidemiology and control of SARS-CoV-2 epidemics in partially vaccinated populations: a modeling study applied to France
}

Paolo Bosetti ${ }^{1 \dagger}$, Cécile Tran Kiem ${ }^{1,2+}$, Alessio Andronico ${ }^{1}$, Vittoria Colizza ${ }^{3}$, Yazdan Yazdanpanah ${ }^{4,5}$, Arnaud Fontanet ${ }^{6,7}$, Daniel Benamouzig ${ }^{8}$ and Simon Cauchemez ${ }^{1 *}$

\begin{abstract}
Background: Vaccination is expected to change the epidemiology and management of SARS-CoV-2 epidemics. Methods: We used an age-stratified compartmental model calibrated to French data to anticipate these changes and determine implications for the control of an autumn epidemic. We assumed vaccines reduce the risk of hospitalization, infection, and transmission if infected by $95 \%, 60 \%$, and $50 \%$, respectively.

Results: In our baseline scenario characterized by basic reproduction number $\mathrm{R}_{0}=5$ and a vaccine coverage of 70 80-90\% among 12-17, 18-59, and $\geq 60$ years old, important stress on healthcare is expected in the absence of measures. Unvaccinated adults $\geq 60$ years old represent $3 \%$ of the population but $43 \%$ of hospitalizations. Given limited vaccine coverage, children aged 0-17 years old represent a third of infections and are responsible for almost half of transmissions. Unvaccinated individuals have a disproportionate contribution to transmission so that measures targeting them may help maximize epidemic control while minimizing costs for society compared to non-targeted approaches. Of all the interventions considered including repeated testing and non-pharmaceutical measures, vaccination of the unvaccinated is the most effective.
\end{abstract}

Conclusions: With the Delta variant, vaccinated individuals are well protected against hospitalization but remain at risk of infection and should therefore apply protective behaviors (e.g., mask-wearing). Targeting non-vaccinated individuals may maximize epidemic control while minimizing costs for society. Vaccinating children protects them from the deleterious effects of non-pharmaceutical measures. Control strategies should account for the changing SARS-CoV-2 epidemiology.

Keywords: SARS-CoV-2, Vaccination, Non-pharmaceutical interventions

\section{Background}

The SARS-CoV-2 pandemic that started in December 2019 has caused more than 5 million deaths around the

\footnotetext{
*Correspondence: simon.cauchemez@pasteur.fr

${ }^{\dagger}$ Paolo Bosetti and Cécile Tran Kiem contributed equally to this work. 'Mathematical Modelling of Infectious Diseases Unit, Institut Pasteur, Université de Paris, UMR2000, CNRS, Paris, France

Full list of author information is available at the end of the article
}

world and led healthcare systems at the brink of collapse in many countries. In addition, the drastic control measures that were implemented to limit its impact have had dramatic socio-economic consequences.

Vaccines have proved effective at reducing the severity of SARS-CoV-2 infection [1], the risk of infection [2], and transmission [3]. A number of modeling studies evaluated how vaccination will help mitigate a SARS-

(c) The Author(s). 2022 Open Access This article is licensed under a Creative Commons Attribution 4.0 International License, which permits use, sharing, adaptation, distribution and reproduction in any medium or format, as long as you give appropriate credit to the original author(s) and the source, provide a link to the Creative Commons licence, and indicate if changes were made. The images or other third party material in this article are included in the article's Creative Commons licence, unless indicated otherwise in a credit line to the material. If material is not included in the article's Creative Commons licence and your intended use is not permitted by statutory regulation or exceeds the permitted use, you will need to obtain permission directly from the copyright holder. To view a copy of this licence, visit http://creativecommons.org/licenses/by/4.0/. The Creative Commons Public Domain Dedication waiver (http://creativecommons.org/publicdomain/zero/1.0/) applies to the data made available in this article, unless otherwise stated in a credit line to the data. 
CoV-2 epidemic rebound this autumn, highlighting that it might be difficult to fully relax control measures given the high transmissibility and severity of SARS-CoV-2 [4-7]. These studies assessed the impact on key health metrics (e.g., number of hospitalizations and death) and identified the level of social distancing that would remain necessary as a function of vaccine coverage. A question that has received less attention is that, in this new era where a large part of the population is vaccinated, the epidemiology of SARS-CoV-2 (Who is infected? Who transmits? Who is hospitalized?) will be different from what it was prior to the distribution of vaccines [8]. It is important to anticipate these changes to determine how control measures might evolve to ensure they maintain the epidemic under control while minimizing costs for society. For example, the expectation that unvaccinated individuals will have a higher contribution to infections, transmissions, and hospitalizations has led some countries to introduce control strategies specifically targeting this population. This is the case of France: confronted to a rapid rise in Delta cases and a plateau in vaccinations in June-July 2021, French authorities announced in July that a sanitary pass, i.e., a proof of completed vaccination, recent infection or recent negative test, would be required to access places such as bars, restaurants, and cinemas. The announcement led to an important surge in vaccination appointments and in vaccine coverage. A number of European countries introduced similar measures.

Here, we developed a mathematical model to characterize the epidemiology of SARS-CoV-2 in a partially vaccinated population and evaluate in this new context the contribution to transmission and healthcare burden of individuals of different ages and vaccination status. This information is used to ascertain different control strategies, including repeated testing and nonpharmaceutical measures, targeting the whole population or subgroups such as unvaccinated individuals to optimally mitigate an autumn epidemic rebound. This is also an opportunity to revisit impact assessment accounting for the increased transmissibility and severity of the Delta variant as well as the reduction in vaccine protection against infection associated with this variant. We consider Metropolitan France as a case study.

\section{Methods}

\section{Deterministic model}

We developed a deterministic age-stratified compartmental model describing the spread of SARS-CoV-2 in metropolitan France. The model, which accounts for French age-specific contact patterns [9], has been described in detail elsewhere [10]. It accounts for a gradient of severity with age [11], assuming that Delta VOC is $50 \%$ more severe than Alpha VOC [12] while Alpha
VOC is $40 \%$ more severe than previously circulating strains [13]. It has been extended to account for the rollout of vaccines [4] as well as the deployment of selfadministered rapid antigenic tests [14]. A full description of the model and equations is reported in the Additional file 1 [15].

\section{Scenarios \\ Vaccine coverages and characteristics}

Considering the Delta variant, we assume that vaccines are $95 \%$ effective at reducing the risk of hospitalization [1], $60 \%$ at reducing the risk of infection [16] (impact on susceptibility), and $50 \%$ at reducing the infectivity of vaccinated individuals [3]. In a sensitivity analysis, we show results if vaccines are $80 \%$ effective at reducing the risk of infection [2] (which was the scenario considered prior to the rise of Delta) and $90 \%$ effective against hospitalization. We build several scenarios regarding vaccine coverage achieved in the different age groups by September 1st, 2021: $90 \%$ or $95 \%$ among those older than 60 years old; $60 \%, 80 \%$, or $90 \%$ among those aged $18-59$ years old and $0 \%, 30 \%$, or $70 \%$ among the $12-17$ years old (called teenagers in the following). To give some context, $89 \%$ of those older than 60 years old, $84 \%$ of the 18-59 years old and $61 \%$ of the $12-17$ years old have received a first dose of vaccines against SARS-CoV2 by August 25th, 2021. In our baseline scenario that we label $70-80-90 \%$, we assume vaccination coverage will reach $70 \%, 80 \%$, and $90 \%$ among $12-17$ years old, $18-59$ years old, and over 60 on September 1st, 2021. In this analysis, we consider that the vaccine coverage corresponds to the proportion of the population having acquired vaccine protection after two doses if required.

\section{Epidemic dynamics with and without control measures}

We assume that, by September 1st, 2021, 25\% (range: $20-30 \%)$ of the French population has been infected by SARS-CoV-2 (see Additional file 1), benefiting from natural protection against reinfection. We then explore scenarios where different types of control measures are implemented.

First, we explore scenarios where control measures are completely relaxed in the Autumn. These scenarios are characterized by the basic reproduction number $R_{0}$, i.e., the average number of persons infected by a case in a population with no immunity and no control measures. In March 2020, $R_{0}$ was estimated at around 3 in France prior to the implementation of a nation-wide lockdown [10]. The emergence of more transmissible variants of concerns (VOC) (such as the Alpha and Delta VOCs) [17-20] is expected to increase $R_{0}$. We therefore explore scenarios in which $R_{0}$ ranges between 3.0 and 6.0 when measures are completely relaxed, considering $R_{0}=5$ as our baseline scenario. We assume that from September 
1st, 2021, the structure of contacts in the population comes back to the one measured during the prepandemic period [9].

We then consider scenarios where different types of control measures are implemented, targeting different groups:

- Random testing: we assume that a proportion of the population is targeted for random testing with antigenic tests. These individuals test at regular intervals (every 7 days in the baseline scenario; twice a week and every 2 weeks in sensitivity analyses). We assume that individuals testing positive isolate in a way that reduces onward transmission by $75 \%$. We consider a scenario where $50 \%$ of unvaccinated individuals aged $\geq 12$ years old get tested and a scenario where the same number of individuals randomly drawn among individuals aged $\geq 12$ years old (vaccinated or unvaccinated) are tested. We consider scenarios where the antigenic test is performed by the individual (self-swabbing and reading of the result; sensitivity: $75 \%$ ) or by a professional (sensitivity 90\%). In a sensitivity analysis, we also explore a scenario where $25 \%$ of unvaccinated individuals $\geq 12$ years old get tested.

- Non-pharmaceutical interventions: Nonpharmaceutical interventions such as social distancing, protective measures and mask-wearing may be used to reduce transmission rates. We consider scenarios where such measures target the whole population, leading to reductions of transmission rates of $10 \%, 20 \%, 30 \%$, or $40 \%$ from any infected individual, whether they have been vaccinated or not. We also consider scenarios where such measures only target unvaccinated individuals, leading to reductions of transmission rates of $10 \%, 20 \%, 30 \%$, or $40 \%$ from unvaccinated individuals, while transmission rates from vaccinated individuals remain unchanged.

- Increased vaccine coverage among unvaccinated individuals: We compare the performance of these interventions to that obtained if $50 \%$ of the unvaccinated individuals aged $\geq 12$ years old were to get vaccinated.

Children are defined as individuals aged 0-17 years old We assume that children aged $0-9$ years old are $50 \%$ less susceptible to infection than adults while those aged 10-17 years old are $25 \%$ less susceptible to infection than adults $[10,21]$. In a sensitivity analysis, we also assume that children aged $0-9$ years old are $50 \%$ less infectious than adults [22].

We assume an antigenic test costs 5 euros if performed by the individual, 11 euros if performed by a professional, and a 2-dose vaccine costs 32 euros. Models are run until March 20th, 2022.

\section{Results}

Baseline scenario and no control measures

We first present results under the assumption that control measures are completely relaxed in autumn 2021, for our baseline scenario (basic reproduction number $R_{0}=5$ and a vaccine coverage of $70-80-90 \%$ among teenagers, adults aged $18-59$ years old and over 60 , respectively). In this case and assuming that $25 \%$ of the population acquired immunity through infection by September 1st, our model anticipates a wave characterized by a peak of 5200 hospital admissions per day which is larger than the peaks observed in France during the two pandemic waves of 2020. The peak would be at 7300 and 3400 admissions per day if the proportion infected by September 1st was $20 \%$ and $30 \%$, respectively.

We anticipate that the roll-out of vaccines will modify the epidemiology of SARS-CoV-2. In a context where most adults are vaccinated but vaccine coverage remains limited among children ( $0-17$ years old), we expect 33\% of infections will occur in this age group, even though they only represent $22 \%$ of the population and are assumed to be less susceptible to SARS-CoV-2 infection than adults (Fig. 1A). In each age group, unvaccinated individuals are overrepresented among infected people while vaccinated individuals are under-represented (Fig. 1B). For example, the risk of infection for an unvaccinated individual is $R R=1.9$ times higher than that of a vaccinated individual among those aged 18-59 years old $(R R=1.3$ among $0-17$ years old and $R R=2.2$ among over 60; Additional file 1: Table 1). Overall, unvaccinated individuals represent $29 \%$ of the population but $46 \%$ of infections. Their contribution to the transmission process is even higher with a risk of transmission from an unvaccinated individual that is 4.3 times higher than that from a vaccinated individual (Fig. 1C, D).

Vaccination will also impact the age distribution of those hospitalized. While $74 \%$ of hospitalizations occurred among those older than 60 years old in the prevaccination era, this proportion is expected to drop to $65 \%$ in our baseline scenario. In parallel, the proportion of 18-59 years old among hospitalized individuals increases from $25 \%$ in the pre-vaccination era to $30 \%$ (Fig. $1 \mathrm{E})$. The small group of unvaccinated adults that are older than 60 years old has a disproportionate impact on the stress to the healthcare system. They represent $10 \%$ of their age group but $66 \%$ of hospitalizations from that age group (RR: 17.2), and 3\% of the general population but $43 \%$ of all hospitalizations (RR: 26.7) (Fig. 1F). Even though we assume that the vaccine is $95 \%$ effective against the risk of hospitalization, in a context where vaccine coverage is high among older individuals, $28 \%$ of 

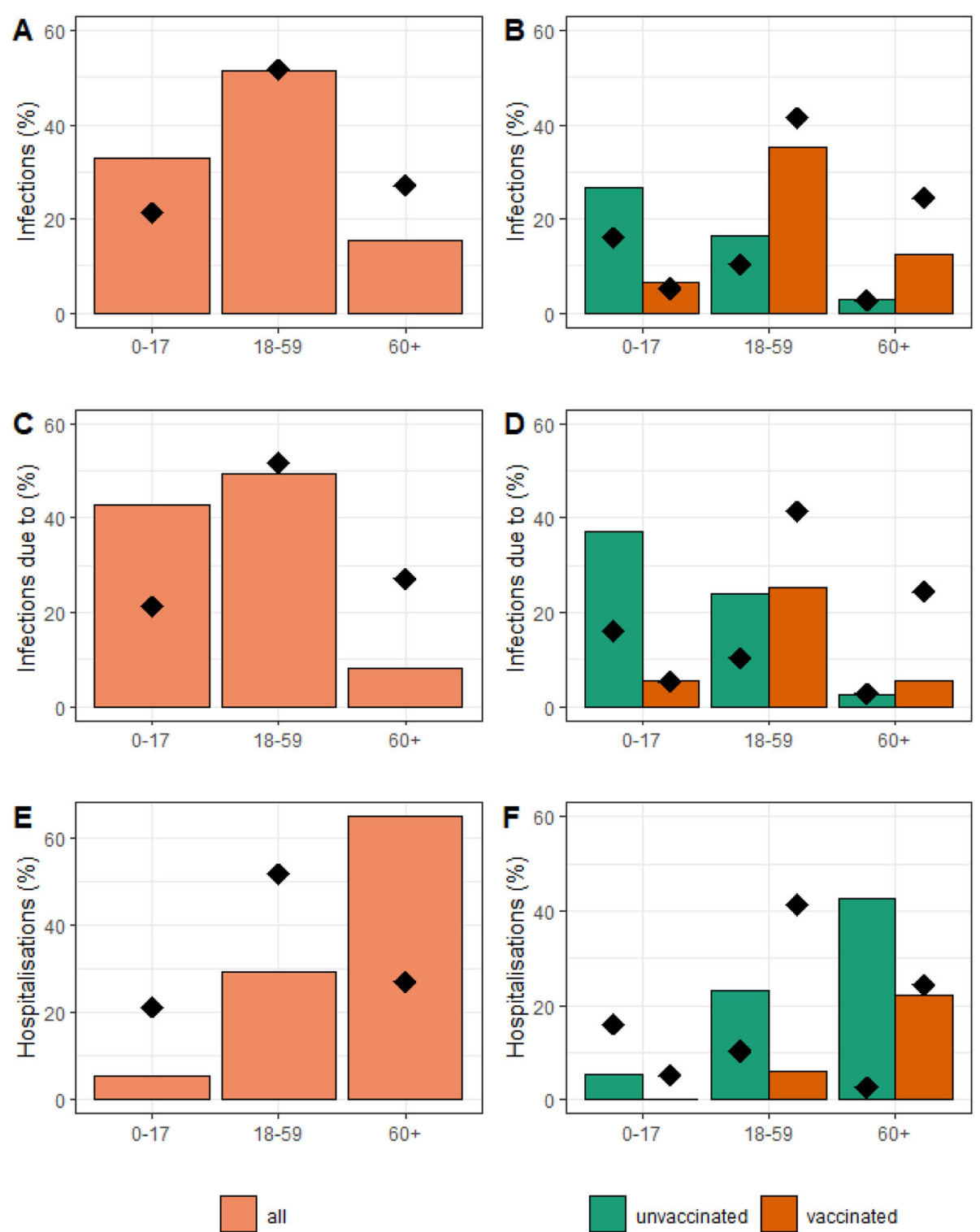

Fig. 1 Contribution of groups defined by their age and vaccination status to infections, disease spread and hospital burden, in our baseline scenario with $R_{0}=5$ and a vaccine coverage of $70-80-90 \%$ among 12-17 years old, 18-59 years old, and over 60 years old. Age distribution of new infections (A) in the entire population and (B) among vaccinated and unvaccinated individuals. Proportion of infections (C) attributable to different age groups and (D) attributable to different age groups among vaccinated and unvaccinated individuals. Age distribution of hospitalizations $(\mathbf{E})$ in the entire population and $(\mathbf{F})$ among vaccinated and unvaccinated individuals. In all panels, the diamonds indicate the age distribution of the different groups in the population

hospitalizations occur among vaccinated people (Fig. $1 \mathrm{~F})$.

\section{Baseline scenario with control measures}

We then investigate the impact of different control strategies targeting different groups for our baseline scenario with a vaccination coverage $70-80-90 \%$ and $R_{0}=5$. Weekly testing of $50 \%$ of unvaccinated individuals aged $\geq 12$ years old could reduce the peak of hospitalizations by $19 \%$ (range: $16-23 \%$ for $20-30 \%$ of the population infected prior to September 1st, 2021) if an autotest is used and 22\% (19-27\%) if the test is performed by a professional (Fig. 2A). In contrast, if the same number of tests were distributed randomly among individuals aged $\geq 12$ years old irrespective of vaccination status, the reductions in hospital admissions would only be of $8 \%$ (7$10 \%)$ and $10 \%(8-12 \%)$, respectively. The reduction in the peak of hospitalizations would be much larger if $50 \%$ of unvaccinated individuals aged $\geq 12$ years old agreed to get vaccinated instead of being repeatedly tested ( $68 \%$ vs 


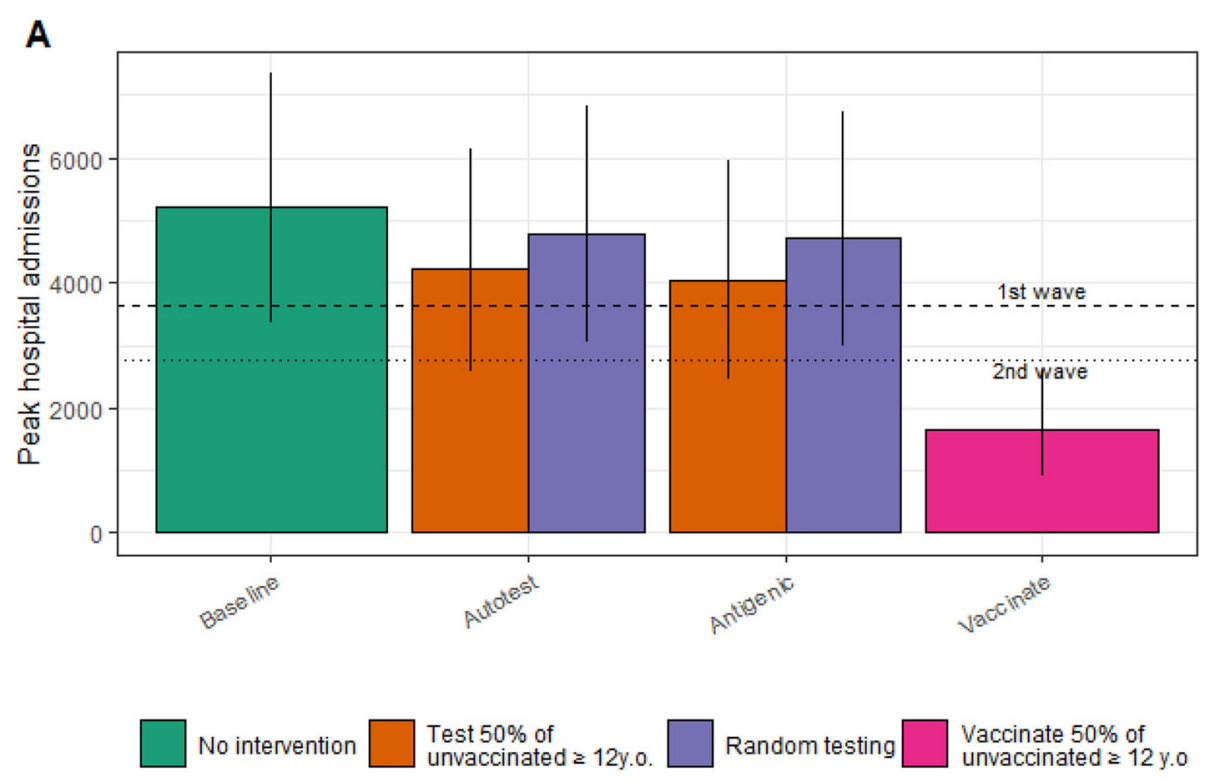

B

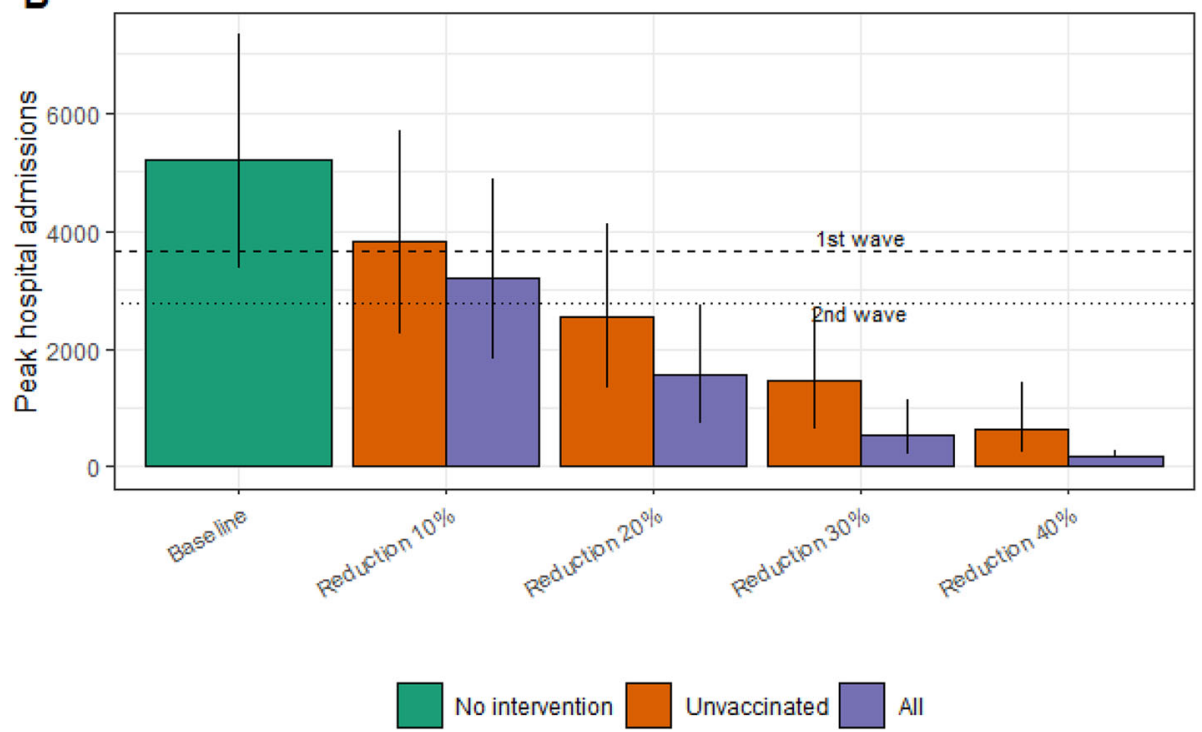

Fig. 2 Comparison of the impact of control strategies targeting the entire population vs unvaccinated individuals only, in our baseline scenario with $R_{0}=5$ and a vaccine coverage of 70-80-90\% among 12-17 years old, 18-59 years old, and over 60 years old. (A) Peak in daily hospital admissions under different testing strategies. Baseline, no intervention; Autotest unvaccinated, 50\% of the unvaccinated individuals aged $\geq 12$ years old are tested weekly (sensitivity of 75\%); Autotest random, the same number of individuals as in the Autotest unvaccinated are tested but among individuals aged $\geq 12$ years old, irrespective of vaccine status; Antigenic unvaccinated, same as in Autotest unvaccinated but with tests performed by a professional (sensitivity of 90\%); Antigenic random, same as in Autotest random but with tests performed by a professional (sensitivity of 90\%); Vaccinate, $50 \%$ of the unvaccinated individuals aged $\geq 12$ years old are vaccinated. $\mathbf{B}$ Peak in daily hospital admissions under nonpharmaceutical interventions of varying intensities. Baseline, no intervention; Reduction of $x \%$ unvaccinated, The transmission rate of unvaccinated individuals is reduced by $x \%$; Reduction of $x \%$ all, The transmission rate at the population level is reduced by $x \%$. We assume $25 \%$ of the population has acquired protection through natural infection (range 20-30\% corresponding to the vertical bars)

19\%; Fig. 2A), for a cost that would be 4.5 times lower (0.16 vs 0.72 billion euros; Additional file 1: Fig. S2). Moreover, only vaccination would be able to reduce the peak of hospital admissions below the peaks observed in the 2020 spring and fall waves.
Non-pharmaceutical interventions applied to all and reducing the overall transmission rates by $10 \%, 20 \%$, $30 \%$, and $40 \%$ would reduce the peak of hospitalizations by $39 \%, 70 \%, 90 \%$, and $97 \%$, respectively (Fig. 2B). If these measures were only targeted towards the few unvaccinated individuals ( $29 \%$ of the population), we could 
still reach $27 \%, 51 \%, 72 \%$, and $87 \%$ peak reductions, respectively. In both scenarios, reducing transmission rates by $20 \%$ would be sufficient to make the peak of hospitalizations drop below the levels observed during the second wave of 2020. Given the residual risk of infection in vaccinated individuals with the Delta variant, substantial gains can be made if vaccinated individuals mitigate their risk of infection for example by wearing masks.

\section{Sensitivity analyses}

Keeping in mind that uncertainties remain about $R_{0}$ and the vaccine coverage in the Autumn, we investigate how our results change if we depart from our baseline assumptions. Figure 3 shows the expected size of the autumn peak in hospital admissions, for different values of $R_{0}$ and vaccine coverages, considering scenarios where control measures can target unvaccinated individuals only or the whole population leading to reductions of transmission rates in the targeted population between $0 \%$ (no control) and $40 \%$. As expected the size of the peak increases with $R_{0}$ and declines with the vaccination coverage. For $R_{0}=3$, which was the value estimated for the historical lineage, we would not anticipate an epidemic rebound for the vaccine coverage expected to be achieved in France. For $R_{0}=4$, the peak is expected to be below the ones of 2020 even if measures are fully relaxed. For $R_{0}=5$, in the absence of control measures, the peak could remain below the one of the autumn 2020, if vaccine coverage was increased to $70 \%$ in teenagers, $90 \%$ in $18-59$ years old, and $95 \%$ in those over 60 years old. For $R_{0}=6$, increasing the vaccine coverage to these levels would still not allow a full relaxation of control measures and the implementation of control measures would be necessary to further mitigate the impact on healthcare. Overall, high vaccination coverage and even limited control of transmission can help mitigate an epidemic rebound. The age distribution of infected and hospitalized individuals depends on vaccine coverage in the different age groups (Fig. 4, Additional file 1: Fig. S3S4-S10-S11). For example, when vaccine coverage in those over 60 years old increases from 90 to $95 \%$, the contribution of this age group to hospitalizations drops from 65 to $55 \%$. Those distributions are relatively robust to a change in $R_{0}$ (Additional file 1: Fig. S3-S4).

Comparing our baseline scenario $(60 \%$ reduction in the risk of infection given Delta) to that with an $80 \%$ reduction in the risk of infection that was considered prior to the rise of Delta, we find that the lower protection conferred by vaccines against Delta infection substantially degrades projections, with a peak of hospitalizations that roughly doubles when moving from 80 to $60 \%$ protection (Fig. 3). This reduction of protection against infection also increases the contribution of vaccinated individuals to infections: they represent $34 \%$ of infections with a protection of $80 \%$ compared to $54 \%$ with a protection of $60 \%$ (Fig. 4, Additional file 1: Fig. S7). As a consequence, compared to vaccinated individuals, unvaccinated individuals are 4 and 10 times more likely to transmit in scenarios with a protection against infection of $60 \%$ and $80 \%$, respectively, for $R_{0}=5$ and a vaccine coverage of $70-80-90 \%$.

For $90 \%$ protection against hospitalization instead of $95 \%$ in our baseline scenario, we expect a $28 \%$ increase in the peak of hospitalizations (Fig. 3). This also increases the proportion of vaccinated individuals among those hospitalized from 28 to $44 \%$ (Fig. 4, Additional file 1: Fig. S8).

If we assume that vaccinated individuals that get infected have the same probability to transmit as unvaccinated individuals (compared to a $50 \%$ reduction in our baseline scenario), the contribution of vaccinated individuals to transmission increases (Additional file 1: Fig. S9). In this scenario, the probability of transmission from an unvaccinated person is 1.7 times higher than that from a vaccinated person (compared to 4.3 with the baseline assumption).

Comparing our baseline scenario (70\% of teenagers vaccinated) to one where teenagers are not vaccinated (Additional file 1: Fig. S12), our results suggest that the vaccination of teenagers may substantially reduce the stress on the healthcare system. For example, if $80 \%$ of $18-59$ years old and $90 \%$ of over 60 are vaccinated, the vaccination of $70 \%$ of teenagers could reduce the peak of hospitalizations by $66 \%$ and $40 \%$ for $R_{0}=4$ and 5 , respectively, compared to a scenario where they are not vaccinated.

If children aged 0-9 years old are 50\% less infectious than adults in addition to being $50 \%$ less susceptible, the proportion of children among infections decreases from 33 to $31 \%$ while the proportion among those that cause infection drops from 43 to $36 \%$ (Additional file 1: Fig. S5). If children aged 0 to 17 years old were as susceptible as adults, the proportion of infections in this age group might reach $44 \%$ (compared to $33 \%$ in the baseline scenario) (Additional file 1: Fig. S6). Furthermore, in our baseline scenario (with vaccine coverage of $70-80-90 \%$ among $12-17,18-59$, and 60 years old and $R_{0}=5$ ), the vaccination of $30 \%$ and $50 \%$ of children aged 5 to 11 years old can reduce the peak in hospital admissions by $20 \%$ and 33\%, respectively (Additional file 1: Fig. S13).

\section{Discussion}

Countries with partially vaccinated populations enter a new era in the control of the SARS-CoV-2 epidemic. However, given the high transmissibility and severity of the Delta variant and the reduced efficacy of vaccines against infection by this variant, SARS-CoV-2 may continue to generate substantial stress on healthcare in the 


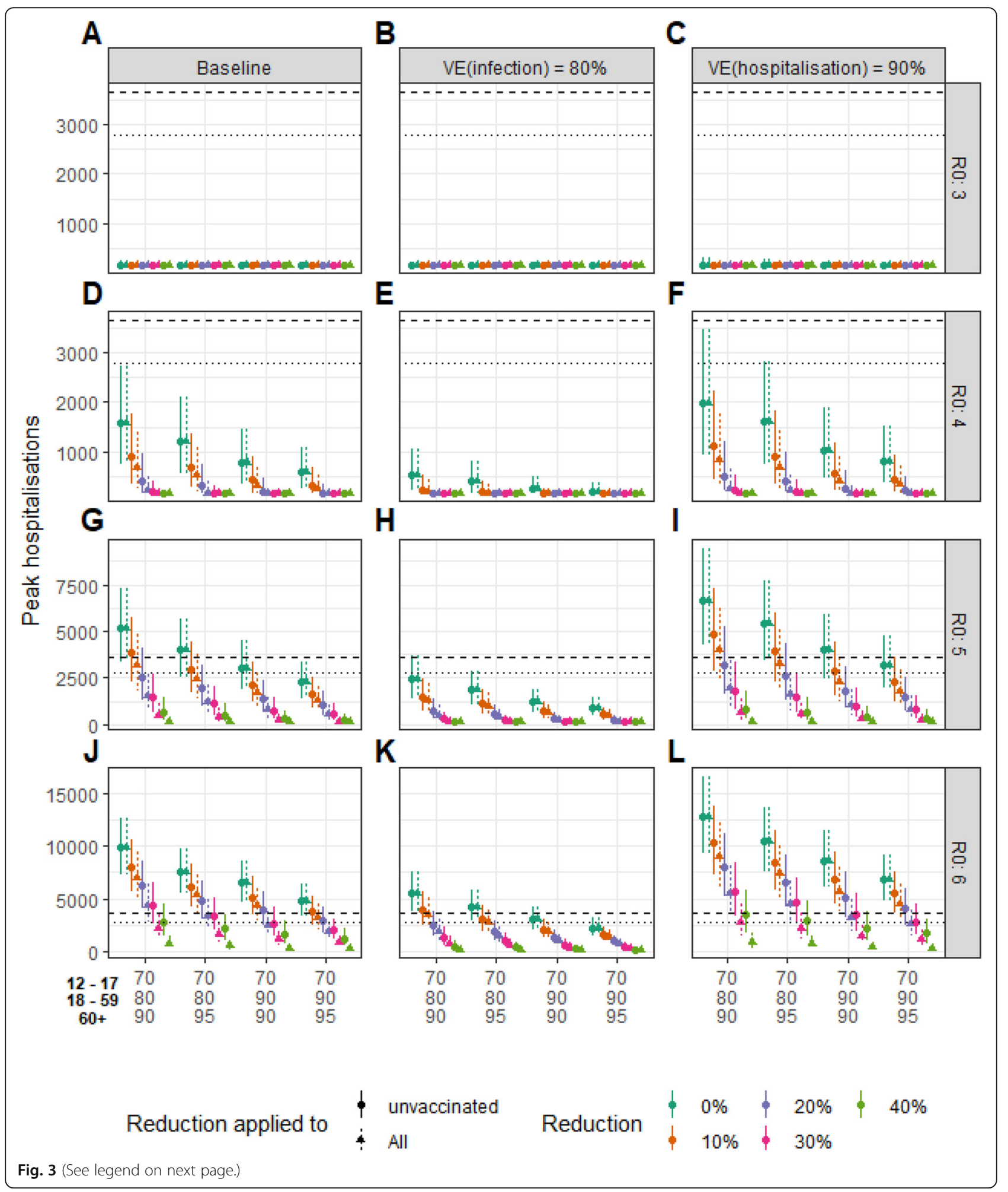

absence of mitigation measures, even with high vaccine coverage. Nonetheless, the partial vaccination of the population modifies the epidemiology of SARS-CoV-2. Here, we used a mathematical model applied to Metropolitan France to anticipate these changes and determine how control measures might evolve in the autumn of 2021 to maximize their impact while minimizing costs.

This autumn, the stress on the healthcare system in the absence of any control measures will depend on the vaccine coverage and the transmission potential $R_{0}$ of 
(See figure on previous page.)

Fig. 3 Expected size of the peak of hospitalizations when non-pharmaceutical interventions target unvaccinated individuals only or the whole population, as a function of the basic reproduction number $R_{0}$, vaccine coverage in the 12-17 years old, 18-59 years old, and over 60 years old and for different efficacy of the vaccine against the risk of infection or hospitalization. Non-pharmaceutical interventions reduce the transmission rate of unvaccinated individuals (points) or the whole population (triangles) by $0 \%, 10 \%, 20 \%, 30 \%$, and $40 \%$. $R_{0}$ takes the values 3.0 (top row, $\mathbf{A}$, B, C), 4.0 ( $\mathbf{D}, \mathbf{E}, \mathbf{F}), 5.0(\mathbf{G}, \mathbf{H}, \mathbf{I})$, and 6.0 (bottom row, J, K, L). In the baseline scenario (left column) we assume that the vaccines are $95 \%$ effective at reducing the risk of hospitalization, $60 \%$ at reducing the risk of infection, and $50 \%$ at reducing the infectivity of vaccinated individuals. In sensitivity analyses, we consider an $80 \%$ reduction against infection (middle column) and a $90 \%$ reduction against hospitalization (right column). We assume $25 \%$ of the population has acquired protection through natural infection (range $20-30 \%$ corresponding to the vertical bars). Horizontal lines indicate the peak of daily hospital admissions observed during the first (dashed line) and the second (dotted line) epidemic wave of 2020

the dominant variant. $R_{0}$ was around 3 for the historical lineages [10]. The Alpha variant was found to be about
$50 \%$ more transmissible than historical lineages [17, 18 , 23 ] and the Delta variant that is now dominant might be
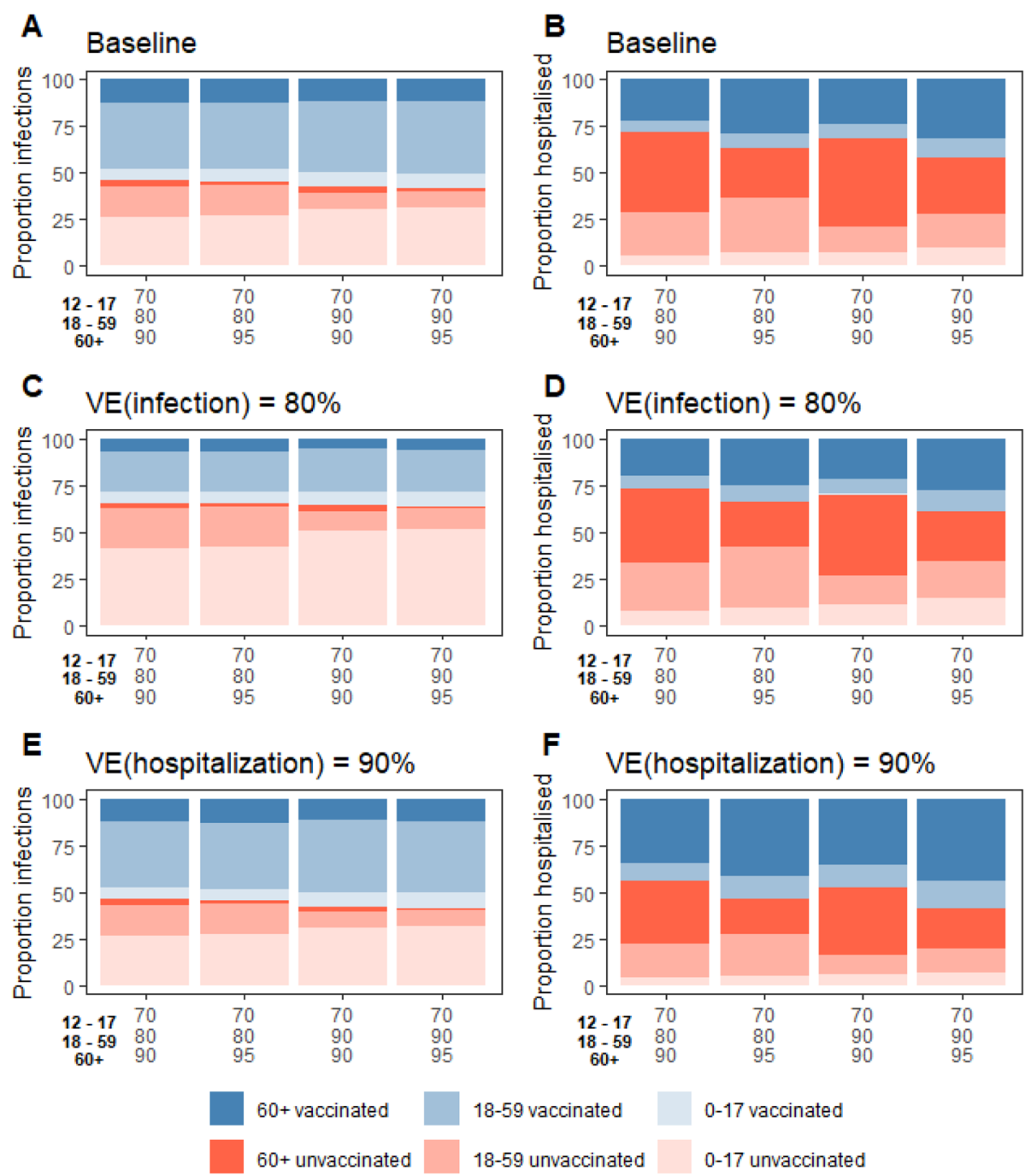

Fig. 4 Proportion of infections (A, C, E) and hospitalizations (B, D, F) among groups defined by their age and vaccination status as a function of the vaccine coverage in the 12-17 years old, 18-59 years old, and over 60 years old. In the baseline scenario (A, B), we assume that vaccines are $95 \%$ effective at reducing the risk of hospitalization, $60 \%$ at reducing the risk of infection and $50 \%$ at reducing the infectivity of vaccinated individuals. In (C and $\mathbf{D})$, we assume a vaccine efficacy at reducing the risk of infection of $80 \%$. In (E and $\mathbf{F})$, we assume a vaccine efficacy at reducing the risk of hospitalization of $90 \%$. The distribution is reported for infections and hospitalizations occurring between September 1 st, 2021, and March 20th, 2022 (end of the study period), for $R_{0}=5.0$. We assume $25 \%$ of the population has acquired protection through natural infection 
more than $50 \%$ more transmissible than the Alpha variant [20]. If we simply apply these multiplicative terms, $R_{0}$ might be as high as 7 for the Delta variant. However, it is possible that transmissibility differences between variants change with control conditions. We therefore considered $R_{0}=5$ in our baseline analysis and explored values between 3 and 6 in our sensitivity analyses. For $R_{0} \geq 5$ which appears likely for the Delta variant and under our baseline vaccine coverage of $70-80-90 \%$ among teenagers, younger and older adults, we anticipate an important stress on the healthcare system in the absence of any control measure (Fig. 3A). Ongoing efforts to control transmission should therefore be maintained. On a more positive note, thanks to vaccination, the intensity of control measures necessary to maintain hospitalizations at manageable levels should be substantially less than what was required before the roll-out of vaccines. Indeed, while lockdowns used in 2020 reduced transmission rates by $70-80 \%$ [10], we find that reductions of the order of $20-30 \%$ might now be sufficient. Such reductions might potentially be achieved through protective measures (e.g., masks, hand hygiene), a certain degree of social distancing, teleworking, the sanitary pass, and Test-Trace-Isolate.

Since vaccines reduce the risk of infection and of transmission if infected, our model anticipates that unvaccinated individuals will contribute more to disease spread than vaccinated ones. Since vaccine coverage among children aged $0-17$ years old will be low relative to that in adults, we anticipate a strong increase of children's contribution, with about one third of infections occurring in children and $43 \%$ being due to this group in our baseline scenario. Adults that are not vaccinated will also disproportionately contribute to the stress on the healthcare system. This is particularly true for those that are older than 60 years old In our baseline scenario, this group represents $3 \%$ of the population but $43 \%$ of hospital admissions.

These observations have important implications for epidemic control. First, they show the importance of obtaining near-perfect vaccine coverages in older age groups that contribute disproportionately to the stress on the healthcare system. This likely requires the development of strategies where authorities reach out to individuals to facilitate their access to vaccines. Second, we anticipate that, in a population that is partially vaccinated, gains achieved thanks to social distancing measures are larger when reducing the contacts of unvaccinated individuals rather than those of vaccinated ones. This suggests that, in this new era, control measures targeting unvaccinated individuals (for example with the use of the sanitary pass) may help maximize epidemic control. Such a targeting strategy raises ethical and social issues. From an economic perspective, targeting unvaccinated individuals maximizes the effectiveness of control while minimizing the cost to society. This is consistent with the theory that in situations where a small group of individuals contributes disproportionately to the spread of disease, it is optimal to target that group. However, targeting the unvaccinated leads to forms of discrimination, felt more or less severely. While it is true that discrimination between the vaccinated and unvaccinated is to some extent the result of individual choices, as vaccines are widely available, these choices are nevertheless socially stratified and correlated with age and socioeconomic status. Moreover, the restrictions put in place are not chosen by individuals but defined by the authorities. Choices may therefore be perceived as discrimination, especially by the unvaccinated. in France, while the sanitary pass has been widely accepted, it has also actively mobilized against it minority segments of the population.

Recent data indicate a reduction in vaccine effectiveness against infection by the Delta variant and a waning of immunity against infection, with protection against hospitalization remaining elevated. These changes have important implications for the management of the epidemic since we expect they will facilitate viral circulation even in highly vaccinated populations and eventually increase the stress on the healthcare system. This means that, compared to the first half of 2021, there is a higher risk that vaccinated individuals get infected and transmit the virus. As a consequence, measures reducing the risk of infection and transmission such as the wearing of the mask should apply to vaccinated individuals in situations where transmission is possible (e.g., indoors).

The situation of children is a particular source of concern. Children aged $<12$ years old do not have access to vaccines yet and vaccine coverage remains lower among teenagers due to later access to the vaccine. While children mostly develop mild SARS-CoV-2 infections, it is essential to secure their access to education and a normal social life and to protect their mental health. Low vaccine coverage among children puts them at risk of being exposed to class closures, with a deleterious impact on their education and mental health [24]. The vaccination of children would insulate them from that risk. In the case of children, the ethical and social problems are exacerbated. On the vaccination side, discrimination arises from the fact that children cannot be seen as making voluntary choices between vaccination and social restrictions. Vaccination is not yet offered under the age of 12 , and beyond the age of 12 , the "choice" to be vaccinated depends primarily on the family environment. As for other measures potentially targeted at schools, a wide range of instruments is available (from mask-wearing to physical distancing, air filtration, iterative self-testing, closing rules, dedicated tracing, isolation of family 
members...) and could help mitigate impact but their targeted implementation would disproportionately affect young people and their families, raising questions of social justice if society at large is less directly targeted, particularly in certain age groups.

This assessment is performed in a context of uncertainty about the value of $R_{0}$ and vaccine coverage in the autumn. Our model makes a number of simplifying assumptions. We ignore a potential decay of immunity, whether immunity was acquired through natural infection or vaccination. Our assumption that vaccination reduces the risk of infection by $60 \%$ represents an average between the value for individuals that have just been vaccinated (who may have higher protection) and that for those that have been vaccinated some time ago (who may have lower protection) [25]. In the absence of boosting, the decay of immunity might cause the model to be too pessimistic for the start of the Autumn wave and too optimistic for the later part of the wave. It could also mean that the contribution of vaccinated individuals to the epidemic process increases progressively as immunity wanes. We also make the assumption that individuals that have been infected remain protected against reinfection, which may lead to optimistic projections. If the decay of immunity is more important among older individuals, we might expect a larger stress on the healthcare system than anticipated in our baseline scenario since older individuals are more likely to develop severe disease if infected. Integrating the decay of immunity and the impact of booster doses in our model is ongoing and will be the subject of future studies. We consider a national model for France and do not account for spatial heterogeneities, that are important [26]. We considered a 'leaky' vaccine that exhibits failure in degree, as most SARS-CoV-2 models [5, 7, 27, 28]. This assumption could lead to larger epidemic sizes than models with "all-or-nothing" vaccines. For this reason, and given the uncertainty on the basic reproductive ratio for Delta, we performed a sensitivity analysis on $R_{0}$.

\section{Conclusion}

We used a mathematical model to anticipate how the epidemiology of SARS-CoV-2 may change in partially vaccinated populations and investigate implications for the control of a possible epidemic rebound this autumn.

\section{Abbreviations}

SARS-CoV-2: severe acute respiratory syndrome coronavirus 2; VOC: Variant of concern; RR: Risk ratio

\section{Supplementary Information}

The online version contains supplementary material available at https://doi. org/10.1186/s12916-022-02235-1.
Additional file 1: Epidemiology and control of SARS-CoV-2 epidemics in partially vaccinated populations: a modeling study applied to France. Table S1: Relative risk of infection, transmission and hospitalization for unvaccinated individuals relative to vaccinated individuals, in different age groups. Figures S1 to S14. Figure S1: Fit to the data. Figure S2: Comparison of the costs of different strategies. Figure S3: Distribution of infections between groups defined by their age and vaccination status. Figure S4: Distribution of hospitalisations between groups defined by their age and vaccination status. Figure S5: Contribution of groups defined by their age and vaccination status to infections, disease spread and hospital burden in a scenario where children aged 0-9 y.o. are 50\% less infectious than adults, in addition to being $50 \%$ less susceptible. Figure S6: Contribution of groups defined by their age and vaccination status to infections, disease spread and hospital burden in a scenario where children aged 0-9 y.o. and teenagers 10-17 are as susceptible as adults. Figure S7: Contribution of groups defined by their age and vaccination status to infections, disease spread and hospital burden in a scenario where the efficacy of the vaccines against infection is set to $80 \%$. Figure S8: Contribution of groups defined by their age and vaccination status to infections, disease spread and hospital burden in a scenario where the efficacy of the vaccines against hospitalisation is set to $\mathbf{9 0 \%}$. Figure S9: Contribution of groups defined by their age and vaccination status to infections, disease spread and hospital burden in a scenario where the vaccinated individuals transmit the virus as the unvaccinated ones. Figure S10: Contribution of groups defined by their age and vaccination status to infections, disease spread and hospital burden, in the scenario with $R_{0}=5$ and a vaccine coverage of $80 \%-90 \%-90 \%$ among $12-17$ y.o., $18-59$ y.o. and over 60 y.o..Figure S11: Contribution of groups defined by their age and vaccination status to infections, disease spread and hospital burden, in the scenario with $\mathrm{RO}=5$ and a vaccine coverage of $80 \%-90 \%-90 \%$ among 12-17 y.o., 18-59 y.o. and over 60 y.o and where the efficacy of the vaccines against hospitalisation is set to $90 \%$. Figure S12: Projections in the absence of control measures, as a function of the basic reproduction number $R_{0}$ and vaccine coverage. Figure S13: Projections in the absence of control measures, as a function of the basic reproduction number $R_{0}$ and different vaccine coverages in children aged 5 to 11 y.o.. Figure S14: Peak in daily hospital admissions under different testing strategies.

\section{Authors' contributions}

PB, CTK, and SC conceived the study. PB, CTK, and AA performed the analyses. PB, CTK, and SC wrote the first draft. VC, YY, AF, and DB contributed to critical revisions of the manuscript. All authors read and approved the final manuscript.

\section{Funding}

We acknowledge financial support from the Investissement d'Avenir program, the Laboratoire d'Excellence Integrative Biology of Emerging Infectious Diseases program (grant ANR-10-LABX-62-IBEID), HAS, Santé Publique France, the EMERGEN project (ANRS0151), the INCEPTION project (PIA/ ANR-16-CONV-0005), the European Union's Horizon 2020 research and innovation program under grant 101003589 (RECOVER) and 874735 (VEO), AXA and Groupama.

\section{Availability of data and materials}

Data and code are available online https://gitlab.pasteur.fr/mmmi-pasteur/ sars-cov-2-epidemics-in-partially-vaccinated-populations.

\section{Declaration}

Ethics approval and consent to participate Not applicable.

Consent for publication Not applicable.

Competing interests

The authors declare that they have no competeing interests. 


\section{Author details}

${ }^{1}$ Mathematical Modelling of Infectious Diseases Unit, Institut Pasteur, Université de Paris, UMR2000, CNRS, Paris, France. ${ }^{2}$ Collège Doctoral, Sorbonne Université, Paris, France. ${ }^{3}$ INSERM, Sorbonne Université, Institut Pierre Louis d'Epidémiologie et de Santé Publique, Paris, France. ${ }^{4}$ Université of Paris, INSERM UMR 1137 IAME, Paris, France. ${ }^{5}$ Department of Infectious Diseases, Assistance Publique-Hôpitaux de Paris, Bichat-Claude-Bernard University Hospital, Paris, France. ${ }^{6}$ Emerging Diseases Epidemiology Unit, Université de Paris, Institut Pasteur, Paris, France. ${ }^{7}$ PACRI Unit, Conservatoire National des Arts et Métiers, Paris, France. ${ }^{8}$ Sciences Po - Centre de sociologie des organisations and Chaire santé - CNRS, Paris, France.

Received: 23 September 2021 Accepted: 4 January 2022

Published online: 26 January 2022

\section{References}

1. Dagan N, Barda N. BNT162b2 mRNA Covid-19 Vaccine in a Nationwide Mass Vaccination Setting. N. Engl. J. Med. 2021;384(15):1412-23. https://doi.org/1 0.1056/NEJMoa2101765.

2. Hall VJ, Foulkes S. COVID-19 vaccine coverage in health-care workers in England and effectiveness of BNT162b2 mRNA vaccine against infection (SIREN): a prospective, multicentre, cohort study. Lancet. 2021;397(10286): 1725-35. https://doi.org/10.1016/S0140-6736(21)00790-X.

3. Harris, R. J.et al. Impact of vaccination on household transmission of SARS COV-2 in England. (2021).

4. Tran Kiem, C.et al. Short and medium-term challenges for COVID-19 vaccination: from prioritisation to the relaxation of measures. (2021).

5. Moore S, Hill EM, Tildesley MJ, Dyson L, Keeling MJ. Vaccination and nonpharmaceutical interventions for COVID-19: a mathematical modelling study. Lancet Infect. Dis. 2021;21(6):793-802. https://doi.org/10.1016/\$1473-3 099(21)00143-2.

6. Viana J, van Dorp CH. Controlling the pandemic during the SARS-CoV-2 vaccination rollout. Nat. Commun. 2021;12(1):3674. https://doi.org/10.1038/ s41467-021-23938-8.

7. Leung K, Wu JT, Leung GM. Effects of adjusting public health, travel, and social measures during the roll-out of COVID-19 vaccination: a modelling study. Lancet Public Health. 2021;6(9):e674-82. https://doi.org/10.1016/S24 68-2667(21)00167-5.

8. Galmiche S, Charmet T. Exposures associated with SARS-CoV-2 infection in France: A nationwide online case-control study. The Lancet Regional Health - Europe. 2021;7:100148. https://doi.org/10.1016/j.lanepe.2021.100148.

9. Béraud G, Kazmercziak S. The French Connection: The First Large Population-Based Contact Survey in France Relevant for the Spread of Infectious Diseases. PLoS One. 2015;10(7):e0133203. https://doi.org/10.1371/ journal.pone.0133203.

10. Salje H, Tran Kiem C. Estimating the burden of SARS-CoV-2 in France. Science. 2020;369(6500):208-11. https://doi.org/10.1126/science.abc3517.

11. Lapidus N, Paireau J. Do not neglect SARS-CoV-2 hospitalization and fatality risks in the middle-aged adult population. Infectious Diseases Now. 2021; 51(4):380-2. https://doi.org/10.1016/j.idnow.2020.12.007.

12. Twohig KA, Nyberg T. Hospital admission and emergency care attendance risk for SARS-CoV-2 delta (B.1.617.2) compared with alpha (B.1.1.7) variants of concern: a cohort study. Lancet Infect. Dis. 2021;22:35-42. https://doi. org/10.1016/S1473-3099(21)00475-8.

13. Bager, P.et al. Increased Risk of Hospitalisation Associated with Infection with SARS-CoV-2 Lineage B.1.1.7 in Denmark. SSRN Electronic Journal (2021) doi:https://doi.org/10.2139/ssrn.3792894

14. Bosetti P, Kiem CT, Yazdanpanah Y, Fontanet A, Lina B, Colizza V, et al. Impact of mass testing during an epidemic rebound of SARS-CoV-2: a modelling study using the example of France. Euro Surveill. 2021;26(1). https://doi.org/10.2807/1560-7917.ES.2020.26.1.2001978.

15. FitzJohn, R. ODE Generation and Integration [R package odin version 1.2.1]. (2021).

16. Tartof, S. Y.et al. Six-Month Effectiveness of BNT162B2 mRNA COVID-19 Vaccine in a Large US Integrated Health System: A Retrospective Cohort Study. (2021) doi:https://doi.org/10.2139/ssrn.3909743.

17. Gaymard A, Bosetti P, Feri A, Destras G, Enouf V, Andronico A, et al. Early assessment of diffusion and possible expansion of SARS-CoV-2 Lineage 201/ 501Y.V1 (B.1.1.7, variant of concern 202012/01) in France, January to March 2021. Euro Surveill. 2021;26(9). https://doi.org/10.2807/1560-7917.ES.2021.26. 9.2100133.
18. Davies, N. G.et al. Estimated transmissibility and impact of SARS-CoV-2 lineage B.1.1.7 in England. Science 372, (2021).

19. Public Health England. Investigation of SARS-CoV-2 variants of concern: variant risk assessments - Risk assessment for SARS-CoV-2 variant: Delta (VOC-21APR-02, B.1.617.2). https://assets.publishing.service.gov.uk/ government/uploads/system/uploads/attachment_data/file/992981/10 June_2021_Risk_assessment_for_SARS-CoV-2_variant_DELTA.pdf (2021).

20. Campbell, F.et al. Increased transmissibility and global spread of SARS-CoV-2 variants of concern as at June 2021. Eurosurveillance vol. 26 (2021).

21. Viner RM, Mytton OT, Bonell C, Melendez-Torres GJ, Ward J, Hudson L, et al. Susceptibility to SARS-CoV-2 Infection Among Children and Adolescents Compared With Adults: A Systematic Review and Meta-analysis. JAMA Pediatr. 2021;175(2):143-56. https://doi.org/10.1001/jamapediatrics.2020.4 573.

22. Li F, Li YY, Liu MJ, Fang LQ, Dean NE, Wong GWK, et al. Household transmission of SARS-CoV-2 and risk factors for susceptibility and infectivity in Wuhan: a retrospective observational study. Lancet Infect. Dis. 2021;21(5): 617-28. https://doi.org/10.1016/S1473-3099(20)30981-6.

23. Volz E, et al. Assessing transmissibility of SARS-CoV-2 lineage B.1.1.7 in England. Nature. 2021;593(7858):266-9. https://doi.org/10.1038/s41586-02103470-x.

24. YoungMinds. Coronavirus: Impact on young people with mental health needs. Survey 4: February 2021. https://youngminds.org.uk/media/4350/ coronavirus-report-winter.pdf (2021).

25. Tartof SY, Slezak JM, Fischer H, Hong V, Ackerson BK, Ranasinghe ON, et al. Effectiveness of mRNA BNT162b2 COVID-19 vaccine up to 6 months in a large integrated health system in the USA: a retrospective cohort study. The Lancet. 2021;398(10309):1407-16. https://doi.org/10.1016/S0140-6736(21)021 83-8.

26. Hozé N, Paireau J, Lapidus N, Tran Kiem C, Salje H, Severi G, et al. Monitoring the proportion of the population infected by SARS-CoV-2 using age-stratified hospitalisation and serological data: a modelling study. Lancet Public Health. 2021;6(6):e408-15. https://doi.org/10.1016/S2468-2667(21 00064-5

27. Hogan $A B$, Winskill P, Watson OJ, Walker PGT, Whittaker C, Baquelin M, et al. Within-country age-based prioritisation, global allocation, and public health impact of a vaccine against SARS-CoV-2: A mathematical modelling analysis. Vaccine. 2021;39(22):2995-3006. https://doi.org/10.1016/j.vaccine.2021.04. 002.

28. Matrajt L, Eaton J, Leung T, Brown ER. Vaccine optimization for COVID-19: Who to vaccinate first? Sci Adv. 2020;7.

\section{Publisher's Note}

Springer Nature remains neutral with regard to jurisdictional claims in published maps and institutional affiliations.

Ready to submit your research? Choose BMC and benefit from:

- fast, convenient online submission

- thorough peer review by experienced researchers in your field

- rapid publication on acceptance

- support for research data, including large and complex data types

- gold Open Access which fosters wider collaboration and increased citations

- maximum visibility for your research: over $100 \mathrm{M}$ website views per year

At $\mathrm{BMC}$, research is always in progress.

Learn more biomedcentral.com/submission 